\title{
A Lifestyle Intervention via Email in Minority Breast Cancer Survivors: Randomized Parallel-Group Feasibility Study
}

Raheem J Paxton ${ }^{1}$, MSc, PhD; Richard Hajek ${ }^{2}, \mathrm{PhD}$; Patricia Newcomb ${ }^{3}, \mathrm{PhD}$; Megha Dobhal ${ }^{4,5}$, MPH; Sujana Borra $^{4,5}$, MPH; Wendell C Taylor ${ }^{6}, \mathrm{MPH}, \mathrm{PhD}$; Deborah Parra-Medina ${ }^{7}, \mathrm{PhD}$; Shine Chang ${ }^{8}$, PhD; Kerry S Courneya ${ }^{9}$, PhD; Gladys Block ${ }^{10,11}$, PhD.; Torin Block ${ }^{10}$, BS; Lovell A Jones ${ }^{12}, \mathrm{PhD}$

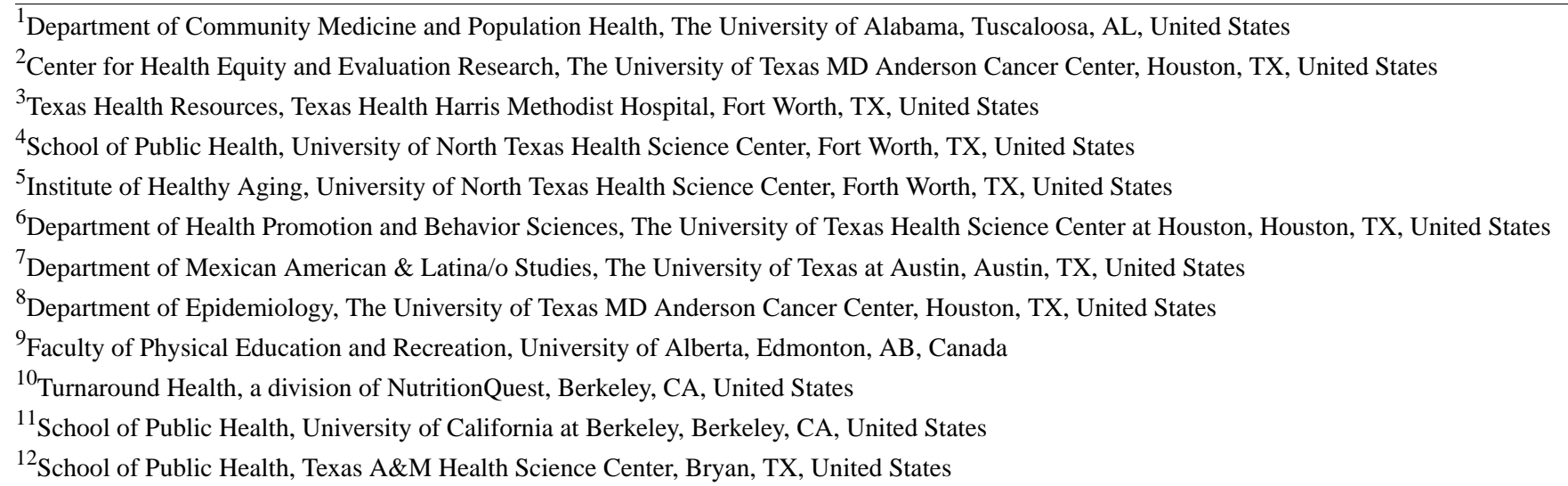

\section{Corresponding Author:}

Raheem J Paxton, MSc, PhD

Department of Community Medicine and Population Health

The University of Alabama

1132 Northeast Medical Bldg

Tuscaloosa, AL, 35401

United States

Phone: 12053486857

Email: rpaxton@ua.edu

\section{Abstract}

Background: Our data have indicated that minority breast cancer survivors are receptive to participating in lifestyle interventions delivered via email or the Web, yet few Web-based studies exist in this population.

Objective: The aim of this study was to examine the feasibility and preliminary results of an email-delivered diet and activity intervention program, "A Lifestyle Intervention Via Email (ALIVE)," delivered to a sample of racial and ethnic minority breast cancer survivors.

Methods: Survivors (mean age: 52 years, 83\% [59/71] African American) were recruited and randomized to receive either the ALIVE program's 3-month physical activity track or its 3-month dietary track. The fully automated system provided tools for self-monitoring and goal setting, tailored content, and automated phone calls. Descriptive statistics and mixed-effects models were computed to examine the outcomes of the study.

Results: Upon completion, 44 of 71 survivors completed the study. Our "intention-to-treat" analysis revealed that participants in the physical activity track made greater improvements in moderate to vigorous activity than those in the dietary track (+97 vs. $+49 \mathrm{~min} /$ week, $P<.001)$. Similarly, reductions in total sedentary time among those in the physical activity track $(-304 \mathrm{vs} .-59$ $\mathrm{min} /$ week, $P<.001$ ) was nearly 5 times greater than that for participants in the dietary track. Our completers case analysis indicated that participants in the dietary track made improvements in the intake of fiber $(+4.4 \mathrm{~g} / \mathrm{day})$, fruits and vegetables $(+1.0$ cup equivalents/day), and reductions in saturated fat $(-2.3 \mathrm{~g} /$ day $)$ and trans fat $(-0.3 \mathrm{~g} /$ day $)($ all $P<.05)$. However, these improvements in dietary intake were not significantly different from the changes observed by participants in the physical activity track (all $P>.05)$. Process evaluation data indicated that most survivors would recommend ALIVE to other cancer survivors (97\%), were 
satisfied with ALIVE (82\%), and felt that ALIVE was effective (73\%). However, survivors expressed concerns about the functionality of the interactive emails.

Conclusions: ALIVE appears to be feasible for racial and ethnic minority cancer survivors and showed promising results for larger implementation. Although survivors favored the educational content, a mobile phone app and interactive emails that work on multiple email domains may help to boost adherence rates and to improve satisfaction with the Web-based platform.

Trial Registration: ClinicalTrials.gov NCT02722850; https://clinicaltrials.gov/ct2/show/NCT02722850 (Archived by WebCite at http://www.webcitation.org/6tHN9VsPh)

(JMIR Cancer 2017;3(2):e13) doi: 10.2196/cancer.7495

\section{KEYWORDS}

breast neoplasm; African Americans; diet; feasibility study; physical activity; posture; program evaluation; Internet; computer tailoring; email

\section{Introduction}

Breast cancer survivors suffer from high rates of overweight or obesity and often do not meet current guidelines for physical activity and intake of fruits and vegetables [1-3]. Poor lifestyle habits of breast cancer survivors contribute to diminished health-related quality of life (HRQoL), increased risk of comorbid conditions, cancer recurrence, and premature mortality [2]. Unfortunately, even though minority breast cancer survivors suffer disproportionately from these circumstances, they remain underserved and underrepresented in epidemiological and intervention research [4-6]. Therefore, studies designed to improve the lifestyle behaviors of minority cancer survivors are warranted.

Comprehensive reviews and meta-analytic studies have indicated that clinic-based or in-person studies intended to improve diet, exercise, and HRQoL in cancer survivors have had promising results [2,7-10]. However, distance and time are fundamental barriers to participating in these studies [3,11]. Several researchers have advocated for home-based interventions that include telephone counseling or tailored print materials $[12,13]$. Whereas many home-based programs have led to significant improvements in healthy lifestyle behaviors [14-20], they are not always sustainable because telephone counseling and mass mailings require significant personnel effort. Studies that utilize the Web offer a potential to overcome the challenges (cost, time, and distance) experienced in traditional home-based interventions [21]. Given these benefits, there has been an increase in advocacy for Web-based interventions [22,23], especially those designed for cancer survivors [24-31]. Previous Web-based studies developed for cancer survivors have observed significant improvements in lifestyle behaviors [28,29,31-34].

Despite the recent surge in Web-based interventions among cancer survivors, few studies have focused on minority cancer survivors [34]. Also, the majority of the studies have focused primarily on physical activity [25-28,30,31], with only a few intervening on dietary intake $[24,29,32]$. Therefore, we proposed to address this limitation by testing the feasibility and preliminary effects of a previously developed fully automated system that utilizes weekly emails, self-monitoring and goal-setting tools, and automated counseling phone calls to improve physical activity and dietary intake [35]. We utilized an evidence-based program entitled "A Lifestyle Intervention
Via Email" (ALIVE) [36]. In previous research, ALIVE demonstrated improvements in moderate to vigorous physical activity and fruit and vegetable consumption as well as reductions in saturated and trans fat in a sample of healthy worksite employees. In this study, participants were randomized to either a physical activity or a dietary track. We hypothesized that survivors randomized to the physical activity track would experience greater improvements in moderate and vigorous physical activity than those randomized to the dietary track. Similarly, we hypothesized that survivors randomized to the dietary track would experience greater improvements in fruit and vegetable consumption and reductions in saturated and trans fats than those randomized to the physical activity track.

\section{Methods}

\section{Recruitment and Consent}

Minority cancer survivors were recruited using nonprobability sampling techniques. Survivors were identified via word of mouth, existing relationships with community-based organizations, and cases ascertained from tumor registries in the North Texas metropolitan area. Eligibility criteria included (1) a previous diagnosis of breast cancer, (2) being at least 18 years old at study enrollment, (3) having completed treatment (except hormonal therapy) at least 6 months before study enrollment, and (4) receptivity to participating in a Web-based intervention study. Also, those who self-identified as African American, Hispanic, or of mixed ethnicity (ie, Asian and African American or African American and non-Hispanic white) were eligible for this study. We used a rolling recruitment process for screening and consenting participants. Survivors completed the screening and consent process from June 2014 to October 2015 using a multi-gated approach. All identified survivors were screened with Web-based surveys that assessed prior history of cancer, lifestyle factors (ie, diet and exercise), and physical activity readiness. The Physical Activity Readiness Questionnaire (PAR-Q) was used to identify contraindications to physical activity[37]. In the event where contraindications were identified, participants were asked to provide information indicating physician approval. Survivors with invalid data or who were not identified as cancer survivors were ineligible. Once survivors completed the screening survey, they were directed to a separate link containing a Web-based consent form. The screening and consent links were distinct from those later delivered for the ALIVE website. Ethical approval by the 
University of North Texas Health Science Center and participating health care institutions was established before enrolling survivors (Clinical trial registration number, NCT02722850).

\section{Randomization and Enrollment}

After participants completed the screening and consent process, a random number generator was used to randomize survivors to either a 3-month physical activity or a 3-month dietary track. Survivors were then sent track-specific enrollment links (ie, physical activity or dietary intake) to begin the ALIVE intervention. Participants in the dietary track could further choose between changing their dietary fat and added sugar intake or their fruit and vegetable intake. Data from participants working on both dietary behaviors were treated as one diet track for this analysis. A total of 71 minority survivors were randomized with equal probability to each track. Survivors received a US \$20 incentive for completing each assessment. Thus, if they completed the baseline and follow-up assessment, they received a total of US $\$ 40$.

\section{Study Goals}

Survivors in the physical activity track were encouraged to meet or exceed current federal recommendations for physical activity (eg, $\geq 150 \mathrm{~min}$ of moderate to vigorous physical activity per week). Survivors in the fruit and vegetable subtrack were encouraged to meet or exceed current recommendations for fruit and vegetable intake (eg, $\geq 3.5$ cup servings of fruit and vegetable consumption). Survivors in the fats and added sugar track were encouraged to decrease intake of saturated and trans fats, decrease added sugars, and increase the intake of "good" fats and carbohydrates to meet or exceed these health recommendations (ie, $\leq 50 \mathrm{~g} /$ day of added sugars and $\leq 10 \%$ of calories from saturated fat) [38]. Content and messages provided to survivors were track specific and designed to promote a target behavior or behaviors.

\section{Intervention}

ALIVE was developed in collaboration between the Kaiser Permanente of Northern California Division of Research and NutritionQuest. No tailoring or modifications were made to the original program for this study. ALIVE was a theory-based coaching system derived from the principles of various theoretical models including the social cognitive theory $[39,40]$, goal-setting theory [41], social marketing [42], and the transtheoretical model [43]. It was designed to enable participants to break up large goals into small achievable goals that could be accomplished weekly. ALIVE was delivered to survivors via an individualized website and interactive emails delivered weekly. At baseline, survivors were asked to complete a diet and activity health risk assessment. The risk assessment provided tailored feedback based on assessed levels of diet and activity and a planning tool to guide improvements in track-specific behaviors. Behavior change strategies such as goal setting, self-monitoring, rewards, cues to action, and repetition were incorporated throughout the program. Functions and features of the ALIVE program were identical across tracks, whereas content (eg, recommended goals and health education materials) differed by track. ALIVE uses participant-reported diet and activity behaviors to individualize the weekly goals it recommended to participants. A brief description of the ALIVE components are reported in Table 1.

\section{Measures}

\section{Physical Activity and Sedentary Behavior}

The Physical Activity Questionnaire (PAQ) was adapted from the Cross-Cultural Activity Participation Study (CAPS) Questionnaire [44]. It comprised 34 domain-specific activities (ie, household and caregiving, sedentary, transportation-related activities, and leisure and sport-related activities). Survivors were asked to indicate how many days per week and minutes per day they participated in each of the activities in a typical week. For the purpose of this study, minutes of moderate to vigorous physical activity per week were utilized as our physical activity outcome. In addition, estimates were derived from several forms of sedentary behavior (ie, total, discretionary, television-viewing, and other), which served as a separate outcome. Test-retest reliability of the instrument utilized in the original ALIVE study indicated adequate reliability [35]. Physical activity and sedentary behaviors were assessed at the baseline and 3-month assessment via the ALIVE system.

\section{Dietary Intake}

The dietary questionnaire queried participants on the intake of 35 commonly consumed foods identified as significant contributors to the intake of fruits and vegetables, added sugars, and saturated and trans fats in the National Health and Nutrition Examination Survey [45]. Survivors were asked to report the frequency and portion size of each of the 35 items and the subtype of select items (eg, diet soda vs non diet soda). The items included commonly consumed foods (eg, hamburgers), fruits and vegetables, nuts, grains (eg, cereals), processed meats (eg, hot dogs), sweets (eg, candy, pastries, and cookies), dairy (eg, milk, eggs, and cheese), and juices (eg, 100\% fruit juice and $\mathrm{Hi}-\mathrm{C}$ ). The response scale ranged from items they consumed multiple times daily to items they consumed only a few times per month. Nutrient estimates were calculated based on consumption patterns and usual portion sizes consumed. The resulting nutrient estimates were derived from established databases [46,47]. The dietary items had acceptable test-retest reliability in the original ALIVE study [35]. Dietary intake was assessed at the baseline and 3-month assessment via the ALIVE system.

\section{Process Evaluation and Feasibility}

Survivors were asked to report on their satisfaction with components of the ALIVE system in a separate Web-based survey. Satisfaction was rated on a 5-point Likert-type response scale ranging from 1 (very dissatisfied) to 5 (very satisfied). We also included a separate overall satisfaction question. We used one question to assess the perceived effectiveness of ALIVE to change health behaviors and another question to assess whether they would recommend ALIVE to other cancer survivors (yes or no). Finally, we included open-ended questions that provided survivors with the opportunity to report on three likes and three dislikes about the ALIVE program. Our process evaluation facilitated our ability to assess the following components of feasibility: acceptability (ie, satisfaction), 
demand (ie, adherence to website usage), implementation and practicality (ie, success or failure of execution reported in the qualitative responses), and limited efficacy (ie, change scores and effect sizes) [48].

\section{Sociodemographic and Medical Data}

These self-report data were collected during the screening survey. The data included items related to age, education, employment status, age at diagnosis, disease stage at diagnosis, and comorbid conditions. We summed the number of comorbid conditions (ie, arthritis, diabetes, high blood pressure, heart disease, and high cholesterol) to create a single continuous variable.

Table 1. Components of the ALIVE (A Lifestyle Intervention Via Email) program by study track.

\begin{tabular}{|c|c|c|}
\hline Features & Physical activity & Dietary intake \\
\hline $\begin{array}{l}\text { Individual tailoring: Information used to } \\
\text { tailor content was based on the baseline diet } \\
\text { and physical activity survey. }\end{array}$ & $\begin{array}{ll}\text { - } & \text { Preference for facility-based or home-based } \\
\text { - } & \text { Stage of physical activity readiness } \\
\text { - } & \text { Social support for exercise } \\
\text { - } & \text { Physical activity barriers } \\
\text { - } & \text { Suggestions to reduce sedentary behavior } \\
\text { - } & \text { User home page }\end{array}$ & $\begin{array}{ll}\text { - } & \text { Habits related to cooking and eating out } \\
\text { - } & \text { Stage of dietary readiness } \\
\text { - } & \text { Specific foods consumed } \\
\text { - } & \text { Social support for healthy eating } \\
\text { - } & \text { Dietary barriers } \\
\text { - } & \text { Suggestions to reduce the top three sources of } \\
& \text { problematic nutrients } \\
\text { - } & \text { User home page }\end{array}$ \\
\hline
\end{tabular}

Tailored goal setting: Content encouraging progress toward goal attainment. New goals were set once old ones were accomplished.

Midweek reminders

Tips: Tips sent out weekly.

Goal tracker: Tracks which goals survivors achieve.

Simulation tool: An interactive feature of the ALIVE website for simulating effects of recommended goals

Health notes: Each week, a different topic was discussed.

Provisions for social support: Weekly goals and tips encouraging survivors to build a support systems with friends and family members. Chat rooms were available for participants to discuss problems and offer solutions to each other.

Automated phone calls: 3- to 5-min calls that facilitated goal setting, provided positive words of encouragement, and emphasized stage specific processes of change. Survivors also queried about personal barriers and goals.
- Weekly emails suggesting four to six smallstep goals tailored to characteristics mentioned above (eg, I will walk $5 \mathrm{~min}$ at lunch time today)

- Queries about physical activity goal achievement

- Reminded survivors of their physical activity goals

- Tips provided information related to achieving physical activity goals and overcoming specific physical activity barriers

- Tracked goals related to the frequency, type, and duration of physical activity

- Allowed the participant to simulate how changing the frequency, quantity, or type of specific activities impacts total physical activity levels

- $\quad$ Topics included research on the relationship between physical activity and various health outcomes

- $\quad$ Provided suggestions such as walks with colleagues at lunch time

- Allowed survivors to engage and troubleshoot physical activity barriers and solutions.
- Weekly emails suggesting four to six smallstep goals tailored to characteristics mentioned above (eg, I will have a salad at lunch one day this week)

Queries about dietary goal achievement

Reminded participants of their dietary goals

Tips provided information related to achieving dietary goals and overcoming specific dietary barriers

- Tracked goals related to the frequency, type, and quantity of dietary nutrients

- Allowed the participant to simulate how changing the frequency, quantity, or type of specific foods or beverages impacts total nutrient levels

- Topics included research on the relationship between a healthy diet and various health outcomes

Provided suggestions to eat healthy meals with friends and family

- Allowed survivors to engage and troubleshoot dietary barriers and solutions.
Calls encouraged:

- Scheduling physical activity

- Overcoming physical activity barriers

- Making public commitments to be active

- Identifying a workout partner

- Reporting your physical activity achievements to others

- Encouraging friends to hold you accountable to activity goals
Calls encouraged:

- Planning healthy meals

- Overcoming dietary barriers

- Making public commitments to consume a healthy diet

- Identifying a friend who would go out and consume a healthy meal with you

- Reporting your dietary achievements to others

- Encouraging friends to hold you accountable to your dietary goals 


\section{Statistical Analysis}

Descriptive statistics were computed to describe the study population. Chi-square tests for independence and Fisher exact tests were used to determine whether there were categorical differences in the sociodemographic and medical variables between study tracks. Subsequent nonparametric Wilcoxon rank-sum tests were computed to determine whether there were mean or median differences in the continuous outcomes at baseline. Generalized mixed-effects models (PROC GLIMMIX) were used to estimate within and between-group changes in study outcomes over time. Given that many of the outcomes were nonnormal, log-normal or Poisson distributions were specified. The effects in the model comprised time, track, time by track interaction, and significant covariates identified in the initial analyses. Furthermore, survivors nested within study tracks were treated as a random effect. Cohen $d$ values were also computed to estimate the effect size. Separate analyses were conducted for cases with complete data and for those where an intention-to-treat (ITT) protocol was applied. To account for missing data in our intention-to-treat analysis, the last observation was carried forward. Furthermore, descriptive statistics were computed for process evaluation data, and $t$ tests were used to make comparisons between the two study tracks. All data were analyzed using Statistical Analysis System (SAS) version 9.3 (SAS Institute Inc, Cary NC), and statistical significance was determined a $P$ value of $\leq .05$ with a two-sided test.

\section{Results}

\section{Descriptive Statistics}

\section{Recruitment and Consent}

In total, 162 minority survivors expressed interest in participating in the study, but only 71 of them $(43.8 \%, 71 / 162)$ received the allocated intervention materials (see Figure 1). Unfortunately, 86 of the 162 persons who expressed interest in the study provided incorrect email addresses $(\mathrm{N}=13)$ or failed to return follow-up emails and phone calls $(\mathrm{N}=73)$. The randomized survivors were on average 52 years old at study enrollment, which was 8 years after initial cancer diagnosis. Most were African American (83\%, 59/71), college educated $(65 \%, 46 / 71)$, and diagnosed with regional stage disease $(54 \%$, $38 / 71)$. Most failed to meet guidelines for intake of fruit and vegetables $(72 \%, 51 / 71)$ and saturated fat $(61 \%, 43 / 71)$. Roughly, half were obese $(52 \%, 37 / 71)$, whereas a surprising number $(63 \%, 45 / 71)$ were already meeting current guidelines for physical activity at baseline (these data are not shown).

Figure 1. Consolidated standards of reporting trials (CONSORT) diagram.

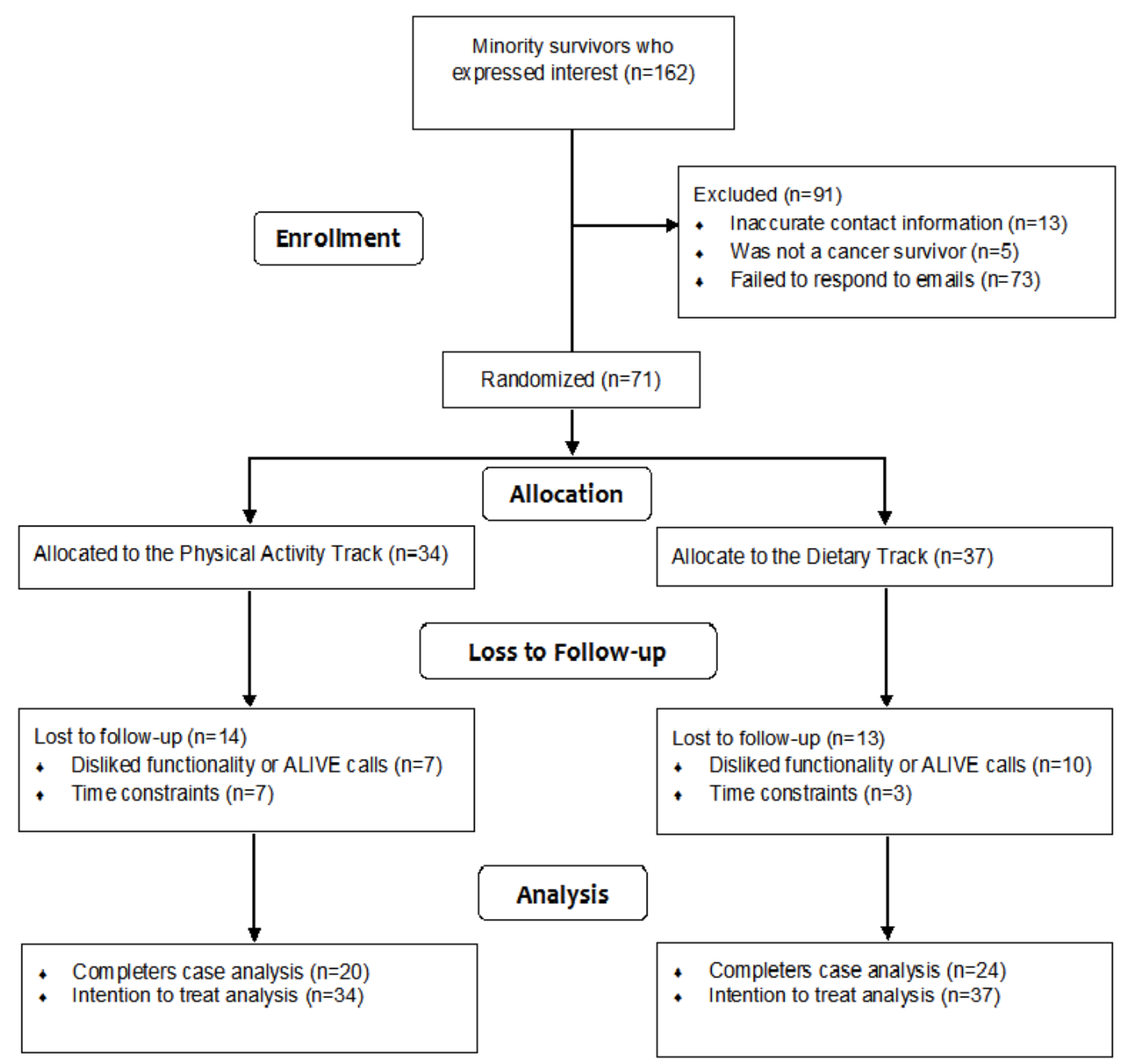


Table 2. Descriptive statistics comparing completers and noncompleters at baseline.

\begin{tabular}{|c|c|c|c|c|}
\hline Variables & $\begin{array}{l}\text { Total sample } \\
\mathrm{N}=71\end{array}$ & $\begin{array}{l}\text { Completers } \\
n=44\end{array}$ & $\begin{array}{l}\text { Noncompleters } \\
\mathrm{n}=27\end{array}$ & $P$ value $^{\mathrm{a}}$ \\
\hline Mean age $\left(\mathbf{S D}^{\mathrm{b}}\right)$ & $52.2(8.6)$ & $52.0(7.8)$ & $52.6(9.9)$ & .62 \\
\hline Median and range of age & $53(26-72)$ & $52(32-69)$ & $54(26-72)$ & - \\
\hline Mean age at diagnosis (SD) & $43.9(8.9)$ & $43.3(7.2)$ & $44.8(11.2)$ & .21 \\
\hline Mean years out from diagnosis (SD) & $8.4(6.5)$ & $8.8(6.9)$ & $7.7(5.8)$ & .57 \\
\hline Race or ethnicity, n (\%) & & & & .86 \\
\hline African American & $59(83)$ & $36(61)$ & $23(39)$ & \\
\hline Hispanic & $8(11)$ & $5(63)$ & $3(37)$ & \\
\hline Mixed & $4(6)$ & $3(75)$ & $1(25)$ & \\
\hline Currently employed, n (\%) & $51(72)$ & $33(75)$ & $18(67)$ & .45 \\
\hline Education, $\mathbf{n}(\%)$ & & & & .20 \\
\hline College graduate & $46(65)$ & $31(70)$ & $15(56)$ & \\
\hline Stage, n (\%) & & & & .60 \\
\hline Localized & $14(20)$ & $9(21)$ & $5(19)$ & \\
\hline Regional & $38(54)$ & $23(52)$ & $15(56)$ & \\
\hline Distant & $19(26)$ & $12(27)$ & $7(25)$ & \\
\hline \multicolumn{5}{|l|}{ Treatment, $\mathbf{n}(\%)$} \\
\hline Surgery & $67(94)$ & $41(93)$ & $26(96)$ & .37 \\
\hline Radiation & $49(69)$ & $30(68)$ & $19(70)$ & .85 \\
\hline Chemotherapy & $53(75)$ & $33(75)$ & $20(74)$ & .93 \\
\hline Hormone & $31(44)$ & $19(43)$ & $12(44)$ & .92 \\
\hline Number of comorbidities, mean (SD) & $0.8(0.9)$ & $0.8(1.1)$ & $0.7(0.7)$ & .93 \\
\hline Median and range of comorbidities & $1(0-4)$ & $1(0-4)$ & $1(0-2)$ & - \\
\hline \multicolumn{5}{|l|}{ Select lifestyle behaviors, mean (SD) } \\
\hline Body mass index & $30.8(6.0)$ & $30.5(5.8)$ & $31.3(6.6)$ & .66 \\
\hline Fruit and vegetable intake in cup servings & $2.8(1.6)$ & $2.7(1.6)$ & $3.0(1.6)$ & .48 \\
\hline Fiber intake in $\mathrm{g} / \mathrm{day}$ & $16.4(8.1)$ & $16.2(7.9)$ & $16.7(8.6)$ & .84 \\
\hline Saturated fat in $\%$ calories & $11.8(7.7)$ & $11.8(7.7)$ & $11.7(7.9)$ & .84 \\
\hline Minutes of moderate to vigorous physical activity/week & $222(272)$ & $240(233)$ & $194(329)$ & .19 \\
\hline Total sedentary minutes/week & $1462(886)$ & $1412(853)$ & $1554(949)$ & .65 \\
\hline
\end{tabular}

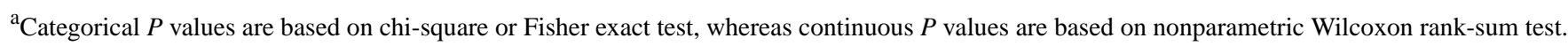
${ }^{\mathrm{b}} \mathrm{SD}$ : standard deviation.

Attrition at the 3-month assessment was 38\% (27/71), with no differences in attrition observed between completers and noncompleters on lifestyle, treatment-related variables, and sociodemographic characteristics (all $P>.05$ ). Descriptive statistics comparing completers and noncompleters are described in Table 2.

\section{Baseline Differences Between Study Tracks}

At the baseline assessment, Hispanic survivors were more likely to be randomized to the physical activity track, and mixed race individuals were more likely to be randomized to the dietary track $(P=.02)$. Descriptive statistics comparing survivors in the diet and physical activity tracks are reported in Table 3. 
Table 3. Descriptive statistics of participants enrolled in ALIVE (A Lifestyle Intervention Via Email) by study tracks at baseline.

\begin{tabular}{|c|c|c|c|}
\hline Variables & $\begin{array}{l}\text { Physical activity } \\
N=34\end{array}$ & $\begin{array}{l}\text { Diet } \\
N=37\end{array}$ & $P$ value $^{\mathrm{a}}$ \\
\hline Dropout, n (\%) & $14(41)$ & $13(35)$ & .60 \\
\hline Mean age $\left(\mathrm{SD}^{\mathrm{b}}\right)$ & $52.7(8.4)$ & $51.8(8.9)$ & .70 \\
\hline Mean age at diagnosis (SD) & $44.6(7.8)$ & $43.3(9.9)$ & .52 \\
\hline Mean years out from diagnosis (SD) & $8.2(5.6)$ & $8.5(7.1)$ & .96 \\
\hline Race or ethnicity, n (\%) & & & .02 \\
\hline African American & $27(79)$ & $32(86)$ & \\
\hline Hispanic & $7(21)$ & $1(3)$ & \\
\hline Mixed or other & $0(0)$ & $4(11)$ & \\
\hline Employment, n (\%) & $26(76)$ & $25(68)$ & .41 \\
\hline \multicolumn{4}{|l|}{ Education, $\mathrm{n}(\%)$} \\
\hline College graduate & $25(74)$ & $21(57)$ & .14 \\
\hline Number of comorbidities, mean (SD) & $0.8(0.8)$ & $0.8(1.1)$ & .57 \\
\hline Stage, n $(\%)$ & & & .16 \\
\hline Localized & $10(29)$ & $4(11)$ & \\
\hline Regional & $16(47)$ & $22(59)$ & \\
\hline Distant & $8(24)$ & $11(30)$ & \\
\hline \multicolumn{4}{|l|}{ Treatment, $\mathbf{n}(\%)$} \\
\hline Surgery & $31(91)$ & $36(97)$ & .34 \\
\hline Radiation & $23(68)$ & $26(70)$ & .81 \\
\hline Chemotherapy & $22(65)$ & $31(84)$ & .07 \\
\hline Hormone & $15(44)$ & $16(43)$ & .94 \\
\hline \multicolumn{4}{|l|}{ Lifestyle behaviors, median $(25 \%-75 \%)^{\mathrm{c}}$} \\
\hline Body mass index & $29.8(25.8-34.1)$ & $31.0(25.8-35.8)$ & .50 \\
\hline Fruit and vegetable intake in cup servings & $2.5(1.4-4.1)$ & $2.8(1.5-3.6)$ & .80 \\
\hline Fiber intake in $\mathrm{g} /$ day & $15.8(10.7-19.7)$ & $15.4(10.2-21.6)$ & .86 \\
\hline Sugar in $\mathrm{g} /$ day & $14.8(7.2-44.5)$ & $24.5(14.1-51.3)$ & .19 \\
\hline Carbohydrates in g/day & $113.7(84.8-197.5)$ & $142.2(106.6-186.0)$ & .28 \\
\hline Trans fat in $\%$ calories & $0.4(0.2-0.8)$ & $0.5(0.3-0.9)$ & .21 \\
\hline Saturated fat in $\%$ calories & $8.8(5.6-13.4)$ & $11.2(6.7-15.1)$ & .14 \\
\hline Minutes of moderate to vigorous physical activity/week & $138(0-390)$ & $150(0-390)$ & $>.99$ \\
\hline Discretionary minutes of sedentary time/week & $1095(660-1680)$ & $1170(510-1860)$ & .93 \\
\hline Other minutes of sedentary time/week & $210(150-720)$ & $360(120-720)$ & .70 \\
\hline Television viewing time/week & $840(420-1260)$ & $720(360-1200)$ & 62 \\
\hline Total sedentary minutes/week & $1410(750-2040)$ & $1380(630-1890)$ & .53 \\
\hline
\end{tabular}

${ }^{a}$ Categorical $P$ values are based on chi-square or Fisher exact test, whereas continuous $P$ values are based on nonparametric Wilcoxon rank-sum test. ${ }^{\mathrm{b}} \mathrm{SD}$ : standard deviation.

${ }^{\mathrm{c}}$ The median and $25 \%$ and $75 \%$ CIs were reported for the lifestyle variables. 


\section{Intervention Outcomes}

\section{Physical Activity}

Our "completers only" and ITT analyses are reported in Tables 4 and 5, respectively. Both tracks made improvements in physical activity (all $P<.001$ ), but the improvements in the physical activity track were greater than that of the dietary track (all $P<.001)$. In particular, the improvements in minutes of moderate physical activity per week were more than twice than that of the dietary track in the completers $(+165$ vs +75 $\mathrm{min} /$ week; $P<.001)$ analysis and nearly two times greater in the ITT (+97 vs +49 min/week; $P<.001)$ analysis.

\section{Sedentary Behavior}

Our analyses indicated that both groups made reductions in discretionary, television-related, and total sedentary time (all $P<.001$ ), but the reductions in the physical activity track were greater than that of the dietary track (all $P<.001$ ). In particular, the reductions in discretionary and television-related sedentary time were more than double than that of the dietary track in both the completers and ITT analyses. More importantly, the reduction in total sedentary time observed among the physical activity track was more than five times ( $-517 \mathrm{vs}-91 \mathrm{~min} /$ week; $P<.001)$ than that of the dietary track in the completers analysis and nearly five times $(-304$ vs $-59 \mathrm{~min} /$ week; $P<.001)$ than that of the dietary track in the ITT analysis.

\section{Dietary Intake}

Our completers case analysis indicates that only the dietary track made improvements in the intake of fiber $(+4.4 \mathrm{~g} /$ day; $P=.01$ ), fruits and vegetables ( +1.0 cup servings/day; $P=.002)$, saturated fat $(-2.8 \mathrm{~g} / \mathrm{day} ; P=.03)$, and trans fat $(-0.3 \mathrm{~g} / \mathrm{day}$; $P=.04)$. In the ITT analysis, only fruit and vegetable intake (+0.7 cup servings/day; $P=.002$ ) improved in the dietary track. The changes observed in our dietary track did not differ from the changes observed in the physical activity track in both the completers case and ITT (all $P>.05$ ) analyses.

Table 4. Change scores for the study outcomes in the completers case analysis ( $\mathrm{N}=44)$.

\begin{tabular}{|c|c|c|c|c|}
\hline Outcomes & $\begin{array}{l}\text { Physical activity } \\
\text { change }^{\mathrm{a}}\left(\mathrm{SE}^{\mathrm{b}}\right) \\
\mathrm{N}=20\end{array}$ & $\begin{array}{l}\text { Dietary intake } \\
\text { change }^{\mathrm{a}}(\mathrm{SE}) \\
\mathrm{N}=24\end{array}$ & Effect size & $P$ value \\
\hline Minutes of moderate to vigorous physical activity/week & $+165(68)^{\mathrm{d}}$ & $+75(62)^{\mathrm{d}}$ & 0.30 & $<.001$ \\
\hline Discretionary minutes of sedentary time/week & $-309(138)^{\mathrm{d}}$ & $-125(126)^{\mathrm{d}}$ & 0.30 & $<.001$ \\
\hline Other minutes of sedentary time/week & $-93(75)^{\mathrm{d}}$ & $+23(68)^{\mathrm{d}}$ & 0.35 & $<.001$ \\
\hline Television viewing time/week & $-216(114)^{\mathrm{d}}$ & $-103(104)^{\mathrm{d}}$ & 0.22 & $<.001$ \\
\hline Total sedentary minutes/week & $-517(148)^{\mathrm{d}}$ & $-91(135)^{\mathrm{d}}$ & 0.64 & $<.001$ \\
\hline Sugar in g/day & $+6.6(4.4)$ & $-2.3(4.0)$ & 0.45 & .43 \\
\hline Fiber in $\mathrm{g} /$ day & $+1.9(1.7)$ & $+4.4(1.6)^{\mathrm{e}}$ & 0.32 & .40 \\
\hline Fruits and vegetables in cup equivalents/day & $+0.6(0.3)$ & $+1.0(0.3)^{\mathrm{d}}$ & 0.28 & .35 \\
\hline Saturated fat in $\mathrm{g} /$ day & $-1.0(1.3)$ & $-0.8(1.2)^{\mathrm{e}}$ & 0.31 & .46 \\
\hline Trans fat in g/day & $+0.0(0.2)$ & $-0.3(0.1)^{\mathrm{e}}$ & 0.51 & .99 \\
\hline Carbohydrates in $\mathrm{g} / \mathrm{day}$ & $+14.2(11.3)$ & $+17.6(10.3)$ & 0.07 & .68 \\
\hline
\end{tabular}

${ }^{a}$ All values represent within-group mean changes for the variables between baseline and follow-up periods.

${ }^{\mathrm{b}} \mathrm{SE}$ : standard error.

${ }^{\mathrm{c}}$ Mixed-effects models were adjusted for race or ethnicity.

${ }^{\mathrm{d}} P<.01$.

${ }^{\mathrm{e}} P<.05$. 
Table 5. Change scores for the study outcomes in the intention-to-treat analysis $(\mathrm{N}=71)$. An intention-to-treat protocol was applied where the last observations were carried forward.

\begin{tabular}{|c|c|c|c|c|}
\hline Outcomes & $\begin{array}{l}\text { Physical activity } \\
\text { change }^{\mathrm{a}}\left(\mathrm{SE}^{\mathrm{b}}\right) \\
\mathrm{N}=34\end{array}$ & $\begin{array}{l}\text { Dietary intake } \\
\text { change }^{\mathrm{a}}(\mathrm{SE}) \\
\mathrm{N}=37\end{array}$ & Effect size & $P$ value \\
\hline Minutes of moderate to vigorous physical activity/week & $+97(42)^{\mathrm{d}}$ & $+49(40)^{\mathrm{d}}$ & 0.20 & $<.001$ \\
\hline Discretionary minutes of sedentary time/week & $-182(85)^{\mathrm{d}}$ & $-81(81)^{\mathrm{d}}$ & 0.20 & $<.001$ \\
\hline Other minutes of sedentary time/week & $-55(45)^{\mathrm{d}}$ & $-15(43)^{\mathrm{e}}$ & 0.15 & $<.001$ \\
\hline Television viewing time/week & $-127(69)^{\mathrm{d}}$ & $-66(67)^{\mathrm{d}}$ & 0.15 & $<.001$ \\
\hline Total sedentary minutes/week & $-304(94)^{\mathrm{d}}$ & $-59(90)^{\mathrm{d}}$ & 0.45 & $<.001$ \\
\hline Sugar in g/day & $+3.9(2.7)$ & $-1.5(2.5)$ & 0.35 & .42 \\
\hline Fiber in g/day & $+1.1(1.1)$ & $+2.9(1.1)$ & 0.27 & .35 \\
\hline Fruits and vegetables in cup equivalents/day & $+0.3(0.2)$ & $+0.7(0.2)^{\mathrm{e}}$ & 0.34 & .29 \\
\hline Saturated fat in $\mathrm{g} /$ day & $-0.6(0.8)$ & $-1.8(0.8)$ & 0.25 & .40 \\
\hline Trans fat in g/day & $-0.0(0.1)$ & $-0.2(0.1)$ & 0.30 & .90 \\
\hline Carbohydrates in $\mathrm{g} / \mathrm{day}$ & $+8.3(6.9)$ & $+11.4(6.6)$ & 0.08 & .61 \\
\hline
\end{tabular}

${ }^{a}$ All values represent within-group mean changes for the variables between baseline and follow-up periods.

${ }^{\mathrm{b}} \mathrm{SE}$ : standard error.

${ }^{\mathrm{c}}$ Mixed-effects models were adjusted for race or ethnicity.

${ }^{\mathrm{d}} P<.01$.

${ }^{\mathrm{e}} P<.05$.

\section{Process Evaluation and Feasibility}

\section{Demand}

Website usage did not differ between study intervention conditions. Survivors in the physical activity track visited the website on an average of 9.6 of the 12 weeks, whereas survivors in the diet track visited the website on an average of 10.7 of the 12 weeks $(P=.15)$.

\section{Satisfaction}

Survivors in both tracks were mostly satisfied with the following components: tips for overcoming barriers, tips for achieving goals, goal-setting tools, and health notes. Additionally, most (97\%) who completed the follow-up assessment indicated that they would recommend the ALIVE program to other cancer survivors. No statistically significant differences were observed between tracks. However, mean scores for the tracking tools were marginally lower in the physical activity track $(P=.05)$. Mean satisfaction scores by track are reported in Table 6.

\section{Implementation and Practicality}

This component of feasibility was assessed via the qualitative responses obtained during our process evaluation. "Likes" reported by survivors could be grouped into six main themes: educational information (36\%), email reminders (14\%), goal-setting tools $(12 \%)$, ease of use $(9 \%)$, and motivation or encouragement $(9 \%)$. The most commonly reported theme related to the educational information presented by the ALIVE program. For example, survivors indicated they liked the "information and tips," and the "Did you know section."

Components of ALIVE that survivors did not like could be grouped into the following themes: Functionality (48\%), information (31\%), tools (14\%), and time (7\%). For functionality, survivors indicated that they "could not enter goals," that "links were not supported" or that they "got stuck" at some point while using the website. Examples of comments pertaining to information were "too much information" and "no relevant patient information."

\section{Limited Efficacy}

The effect sizes measuring changes in dietary intake between tracks were mostly medium in size. In the completers case analysis (see Table 4), effect sizes ranged from 0.28 for fruit and vegetable intake to 0.45 for sugar intake. In the ITT analysis (see Table 5), effect sizes were more modest but similar in magnitude (range $=0.25$ for saturated fat intake to 0.35 for added sugar intake). The effect sizes measuring changes in physical activity and sedentary behavior between tracks differed by the variable of interest and analysis. In both the completers case and ITT analysis, the effect sizes were small for television viewing ( 0.22 for completers case and 0.15 for ITT analysis). However, for total sedentary time, the effect sizes were mostly large ( 0.64 for completers case and 0.45 for ITT analysis). For physical activity, the effect sizes were small $(0.20)$ for the ITT analysis but medium for the completers case analysis (0.30). 
Table 6. Process evaluation data for study participants.

\begin{tabular}{|c|c|c|c|c|}
\hline Satisfaction ( $1=$ not at all, $5=$ very satisfied $)$ & Total & Physical activity & Diet & $P$ value $^{\mathrm{a}}$ \\
\hline Tips for overcoming barriers & $4.2(0.6)$ & $4.1(0.7)$ & $4.2(0.6)$ & .63 \\
\hline Tips for achieving goals & $4.2(0.6)$ & $4.2(0.7)$ & $4.3(0.6)$ & .78 \\
\hline Tracker of daily habits & $3.7(0.8)$ & $3.4(0.8)$ & $4.0(0.8)$ & .05 \\
\hline Progress tools - tracks current and past goals & $3.9(0.9)$ & $3.6(1.0)$ & $4.2(0.7)$ & .08 \\
\hline Simulator tools- tool to help you visualize success & $4.0(0.7)$ & $4.0(0.7)$ & $4.0(0.6)$ & .99 \\
\hline Goal-setting tools & $4.2(0.7)$ & $4.3(0.7)$ & $4.1(0.8)$ & .46 \\
\hline Automated phone coaching & $3.5(1.3)$ & $3.4(1.2)$ & $3.6(1.3)$ & .68 \\
\hline Tailored newsletters & $4.0(0.9)$ & $4.1(0.8)$ & $3.9(1.0)$ & .57 \\
\hline Health note—articles to increase knowledge and skills & $4.2(0.9)$ & $4.2(0.8)$ & $4.1(1.0)$ & .85 \\
\hline Overall satisfaction & $4.1(0.9)$ & $3.9(1.0)$ & $4.3(0.7)$ & .24 \\
\hline Effectiveness in changing behavior ( $1=$ not at all, $5=$ very effective) & $3.8(0.9)$ & $3.7(1.1)$ & $3.8(0.7)$ & .67 \\
\hline Recommend ALIVE ${ }^{\mathrm{b}}$ to other survivors, $\%$ yes & 97 & 95 & 100 & .47 \\
\hline
\end{tabular}

${ }^{\mathrm{a}} t$ tests were used to compare continuous indicators, and chi-square test of independence were used to compare the single binary item.

${ }^{b}$ ALIVE: A Lifestyle Intervention Via Email.

\section{Discussion}

\section{Principal Findings}

In this randomized parallel-group study, we observed that survivors randomized to the physical activity track made greater improvements in physical activity and greater reductions in sedentary behavior than those randomized to the dietary track. Despite the improvements in our activity-related constructs, these data only partially support our initial hypotheses, given that changes in the dietary variables did not differ significantly between tracks. Our process evaluation indicated that survivors were mostly satisfied with ALIVE and would recommend it to other survivors. However, concerns about ALIVE were noted. Overall, these data demonstrate that Web-based interventions such as ALIVE are feasible for racial and ethnic minority breast cancer survivors, but challenges must be addressed to improve the end user experience. The Alive program developers have recently developed and tested an updated version of the program that addresses some of the concerns identified in this study.

This is one of the first studies to examine the feasibility of a fully automated Web-based intervention in a sample of underserved breast cancer survivors. Our feasibility data were favorable, but attrition rates were high. The study's attrition rate was comparable to previous Web-based intervention studies [49-51] but higher than recent studies conducted among cancer survivors [24,26,29-31,34]. Our team discovered that functionality challenges contributed to high attrition rates. Challenges reported by survivors included repeat calls from the automated phone system and ALIVE email messages not being fully interactive within certain email domains (ie, AOL, Thunderbird, Live, Outlook, and Lotus) nor on mobile phones or tablets. Therefore, many survivors were only able to access ALIVE from a desktop computer. The challenges resulted in considerable frustration and many asked to be removed from the study. Unfortunately, our team was not aware of the technical difficulties before the study. However, we worked with NutritionQuest to address the challenges and identify solutions for participants. Encouragingly, our process evaluation was overwhelmingly positive, despite the challenges.

ALIVE was associated with significant improvements in physical activity for both tracks. Prior Web-based interventions among cancer survivors have observed significant improvements in physical activity [24,28-31,33,34], which ranged from 18 min [24] to 103 min [30]. Importantly, in our physical activity track, we observed a 165-min increase in our completers analysis and a 97-min increase in our ITT analysis. Despite these broad improvements, our effect sizes were small to medium in magnitude. The small effect sizes may be due to transfer effects [52], whereby setting goals in one's behavior increases one's confidence, intentions, and motivation to make improvements in another behavior [53-55]. Here, setting goals for diet may have transferred over to physical activity. Transfer effects may be common among cancer survivors because they capitalize on the "teachable moment" following their cancer diagnosis.

To our knowledge, this is one of the first Web-based studies among cancer survivors to observe significant changes in sedentary time. ALIVE was not designed to be a sedentary behavior intervention, yet reductions in sedentary time were observed among our physical activity track. In discussions with NutritionQuest to inquire about the sedentary behavior curriculum, we were informed that educational materials discussing sedentary behaviors were minimal. Observed improvements in sedentary activity could be the result of this minimal sedentary behavior program content. Alternatively, it could be a transfer effect, similar to what was observed in dietary track. More research is needed to determine how, when, and for whom transfer effects occur.

Few Web-based interventions for cancer survivors have intervened on dietary intake. Our completers case analysis indicated significant improvements in the intake of fiber, fruits 
and vegetables intake, and saturated fat for the dietary track. These data support the results found in the original ALIVE study [36]. However, the observed changes did not differ significantly between tracks. Additionally, similar results were not observed in our ITT analysis. To our knowledge, only three Web-based intervention studies among cancer survivors have intervened on dietary intake [24,29,32], with two studies showing improvements $[29,33]$. It could be that 3 months were not sufficient to produce changes in dietary intake in our sample. Recently, Kanera et al [33] demonstrated an improvement in dietary intake at 12 months; a diet effect was not significant at the 6-month assessment [32]. More research is needed to determine the recommended program length required to change behavioral outcomes in Web-based intervention studies.

\section{Limitations}

Our study has limitations. Our team used a convenient sampling strategy to maximize our recruitment efforts, and our sample consisted mostly of African American survivors who were college educated. It should also be noted that eligibility was not based on baseline physical activity or dietary behaviors. In particular, some participants were meeting guidelines for physical activity or dietary intake before joining the study. This may have lowered our estimated effect size between study tracks. Prior studies have observed stronger effects among survivors not meeting guidelines to lifestyle behaviors at the baseline assessment [50]. Furthermore, our attrition rate was high, and many survivors did not return our emails or calls lowering our accrual rate. We are uncertain why participants never returned our emails or calls. Our team can only speculate that our emails with embedded links to the ALIVE websites were identified as junk mail and never received by the survivors. Other survivors who failed to complete the study were either not sufficiently engaged, were frustrated by technical challenges, or had competing priorities that reduced their interest in completing the study. Finally, our outcome measures were self-report and subject to recall and reporting biases. Self-report surveys are common in Web-based interventions, where obtaining objective estimates of physical activity and dietary intake would be costly. Despite the limitations, there are several strengths, including (1) a focus on high-priority breast cancer survivors, (2) significant or positive trends in lifestyle behavior changes, and (3) use of an evidence-based intervention tool with demonstrated efficacy in healthy adults.

\section{Conclusions}

ALIVE appears to be feasible for racial and ethnic minority breast cancer survivors and capable of improving multiple lifestyle behaviors. Although we observed favorable ratings for ALIVE, improvements to functionality and a tailoring to cancer survivors are warranted. Web-based programs should be created to minimize challenges that the end user would encounter. Since the time our study concluded, the developers of ALIVE have released an updated version of the program that includes features to increase engagement and reduce attrition. In particular, the newest version of ALIVE was designed to operate on phones, tablets, and computers; includes a stand-alone mobile phone app; and uses gamification, a points system, and other strategies to increase adherence [56]. Additional studies are needed to test the platform utilized here as well as the newest version of ALIVE in a sample of breast cancer survivors. Such studies could recruit a larger and more ethnically diverse sample to explore similarities and differences in the adoption and maintenance of lifestyle behaviors. Fully automated programs such as ALIVE are capable of being incorporated with minimal cost in clinical and community-based settings with the potential for dissemination and implementation to thousands of cancer survivors nationwide.

\section{Acknowledgments}

The authors would like to thank several community-based organizations, including the Sisters Network Inc, Army of Women, Bridge Breast Network, and Cancer Care Services for assisting us in recruiting racial and ethnic minority breast cancer survivors. Importantly, they would like to thank the women who continue to fight against breast cancer; may this work help the struggle. The authors would also like to thank Ms Susan Page for providing editorial assistance. This research was supported, in part, by National Cancer Institute grants K01CA158000 (RJP).

\section{Authors' Contributions}

$\mathrm{RP}, \mathrm{RH}$, and PN spearhead recruitment efforts and IRB protocols at the respective institutions. RP, WC, SC, LJ, and KC conceived the study. RP, SB, and MD participated in tracking and tracing participants and entering data. All authors participated in drafting and editing the manuscript.

\section{Conflicts of Interest}

GB and TB hold the copyright on ALIVE and have financial interest in ALIVE.

\section{Multimedia Appendix 1 \\ CONSORT-EHEALTH checklist (v1.6.1).}

[PDF File (Adobe PDF File), 548KB-Multimedia Appendix 1]

\section{References}


1. Blanchard CM, Courneya KS, Stein K, American Cancer Society's SCS-II. Cancer survivors' adherence to lifestyle behavior recommendations and associations with health-related quality of life: results from the American Cancer Society's SCS-II. J Clin Oncol 2008 May 1;26(13):2198-2204 [FREE Full text] [doi: 10.1200/JCO.2007.14.6217] [Medline: 18445845]

2. Rock CL, Doyle C, Demark-Wahnefried W, Meyerhardt J, Courneya KS, Schwartz AL, et al. Nutrition and physical activity guidelines for cancer survivors. CA Cancer J Clin 2012;62(4):243-274 [FREE Full text] [doi: 10.3322/caac.21142] [Medline: 22539238]

3. Demark-Wahnefried W, Peterson B, McBride C, Lipkus I, Clipp E. Current health behaviors and readiness to pursue life-style changes among men and women diagnosed with early stage prostate and breast carcinomas. Cancer 2000 Feb 1;88(3):674-684. [Medline: 10649263]

4. Adams SA, Butler WM, Fulton J, Heiney SP, Williams EM, Delage AF, et al. Racial disparities in breast cancer mortality in a multiethnic cohort in the Southeast. Cancer 2012 May 15;118(10):2693-2699 [FREE Full text] [doi: 10.1002/cncr.26570] [Medline: 21953316]

5. Leopold C, Wagner AK, Zhang F, Lu CY, Earle C, Nekhlyudov L, et al. Racial disparities in all-cause mortality among younger commercially insured women with incident metastatic breast cancer. Breast Cancer Res Treat 2016 Jul;158(2):333-340. [doi: 10.1007/s 10549-016-3875-z] [Medline: 27342456]

6. Samson ME, Adams SA, Orekoya O, Hebert JR. Understanding the association of type 2 diabetes mellitus in breast cancer among African American and European American populations in South Carolina. J Racial Ethn Health Disparities 2016 Sep;3(3):546-554. [doi: 10.1007/s40615-015-0173-0] [Medline: 27294746]

7. Schmitz KH, Holtzman J, Courneya KS, Mâsse LC, Duval S, Kane R. Controlled physical activity trials in cancer survivors: a systematic review and meta-analysis. Cancer Epidemiol Biomarkers Prev 2005 Jul;14(7):1588-1595 [FREE Full text] [doi: 10.1158/1055-9965.EPI-04-0703] [Medline: 16030088]

8. Speck RM, Courneya KS, Mâsse LC, Duval S, Schmitz KH. An update of controlled physical activity trials in cancer survivors: a systematic review and meta-analysis. J Cancer Surviv 2010 Jun;4(2):87-100. [doi: 10.1007/s11764-009-0110-5] [Medline: 20052559]

9. Focht BC, Clinton SK, Devor ST, Garver MJ, Lucas AR, Thomas-Ahner JM, et al. Resistance exercise interventions during and following cancer treatment: a systematic review. J Support Oncol 2013 Jun;11(2):45-60. [Medline: 23967493]

10. Rock CL, Demark-Wahnefried W. Nutrition and survival after the diagnosis of breast cancer: a review of the evidence. $\mathrm{J}$ Clin Oncol 2002 Aug 01;20(15):3302-3316 [FREE Full text] [doi: 10.1200/JCO.2002.03.008] [Medline: 12149305]

11. Andajani-Sutjahjo S, Ball K, Warren N, Inglis V, Crawford D. Perceived personal, social and environmental barriers to weight maintenance among young women: a community survey. Int J Behav Nutr Phys Act 2004 Dec 5;1(1):15 [FREE Full text] [doi: 10.1186/1479-5868-1-15] [Medline: 15462679$]$

12. Goode AD, Reeves MM, Eakin EG. Telephone-delivered interventions for physical activity and dietary behavior change: an updated systematic review. Am J Prev Med 2012 Jan;42(1):81-88. [doi: 10.1016/j.amepre.2011.08.025] [Medline: 22176852]

13. Eakin EG, Lawler SP, Vandelanotte C, Owen N. Telephone interventions for physical activity and dietary behavior change: a systematic review. Am J Prev Med 2007 May;32(5):419-434. [doi: 10.1016/j.amepre.2007.01.004] [Medline: 17478269]

14. Demark-Wahnefried W, Clipp EC, Morey MC, Pieper CF, Sloane R, Snyder DC, et al. Lifestyle intervention development study to improve physical function in older adults with cancer: outcomes from Project LEAD. J Clin Oncol 2006 Jul 20;24(21):3465-3473 [FREE Full text] [doi: 10.1200/JCO.2006.05.7224] [Medline: 16849763]

15. Goodwin PJ, Segal RJ, Vallis M, Ligibel JA, Pond GR, Robidoux A, et al. Randomized trial of a telephone-based weight loss intervention in postmenopausal women with breast cancer receiving letrozole: the LISA trial. J Clin Oncol 2014 Jul 20;32(21):2231-2239. [doi: 10.1200/JCO.2013.53.1517] [Medline: 24934783]

16. Grimmett C, Simon A, Lawson V, Wardle J. Diet and physical activity intervention in colorectal cancer survivors: a feasibility study. Eur J Oncol Nurs 2015 Feb;19(1):1-6 [FREE Full text] [doi: 10.1016/j.ejon.2014.08.006] [Medline: 25245710]

17. Harris MN, Swift DL, Myers VH, Earnest CP, Johannsen NM, Champagne CM, et al. Cancer survival through lifestyle change (CASTLE): a pilot study of weight loss. Int J Behav Med 2013 Sep;20(3):403-412. [doi: 10.1007/s12529-012-9234-5] [Medline: 22535636]

18. Hawkes AL, Chambers SK, Pakenham KI, Patrao TA, Baade PD, Lynch BM, et al. Effects of a telephone-delivered multiple health behavior change intervention (CanChange) on health and behavioral outcomes in survivors of colorectal cancer: a randomized controlled trial. J Clin Oncol 2013 Jun 20;31(18):2313-2321. [doi: 10.1200/JCO.2012.45.5873] [Medline: 23690410]

19. Morey MC, Snyder DC, Sloane R, Cohen HJ, Peterson B, Hartman TJ, et al. Effects of home-based diet and exercise on functional outcomes among older, overweight long-term cancer survivors: RENEW: a randomized controlled trial. J Am Med Assoc 2009 May 13;301(18):1883-1891 [FREE Full text] [doi: 10.1001/jama.2009.643] [Medline: 19436015]

20. Rock CL, Byers TE, Colditz GA, Demark-Wahnefried W, Ganz PA, Wolin KY, Exercise and Nutrition to Enhance Recovery and Good Health for You (ENERGY) Trial Group. Reducing breast cancer recurrence with weight loss, a vanguard trial: the Exercise and Nutrition to Enhance Recovery and Good Health for You (ENERGY) Trial. Contemp Clin Trials 2013 Mar;34(2):282-295 [FREE Full text] [doi: 10.1016/j.cct.2012.12.003] [Medline: 23266440] 
21. Goode AD, Lawler SP, Brakenridge CL, Reeves MM, Eakin EG. Telephone, print, and web-based interventions for physical activity, diet, and weight control among cancer survivors: a systematic review. J Cancer Surviv 2015 Mar 11. [doi: 10.1007/s11764-015-0442-2] [Medline: 25757733]

22. Paxton RJ, Nayak P, Taylor WC, Chang S, Courneya KS, Schover L, et al. African-American breast cancer survivors' preferences for various types of physical activity interventions: a Sisters Network Inc. web-based survey. J Cancer Surviv 2014 Mar;8(1):31-38 [FREE Full text] [doi: 10.1007/s11764-013-0307-5] [Medline: 24043292]

23. Badr H, Chandra J, Paxton RJ, Ater JL, Urbauer D, Cruz CS, et al. Health-related quality of life, lifestyle behaviors, and intervention preferences of survivors of childhood cancer. J Cancer Surviv 2013 Dec;7(4):523-534 [FREE Full text] [doi: 10.1007/s11764-013-0289-3] [Medline: 23749663]

24. Bantum EO, Albright CL, White KK, Berenberg JL, Layi G, Ritter PL, et al. Surviving and thriving with cancer using a Web-based health behavior change intervention: randomized controlled trial. J Med Internet Res 2014;16(2):e54 [FREE Full text] [doi: 10.2196/jmir.3020] [Medline: 24566820]

25. De Cocker K, Charlier C, Van Hoof E, Pauwels E, Lechner L, Bourgois J, et al. Development and usability of a computer-tailored pedometer-based physical activity advice for breast cancer survivors. Eur J Cancer Care (Engl) 2015 Sep;24(5):673-682. [doi: 10.1111/ecc.12225]

26. Forbes CC, Blanchard CM, Mummery WK, Courneya KS. Feasibility and preliminary efficacy of an online intervention to increase physical activity in Nova Scotian cancer survivors: a randomized controlled trial. JMIR Cancer 2015 Nov 23;1(2):e12 [FREE Full text] [doi: 10.2196/cancer.4586] [Medline: 28410166]

27. Frensham LJ, Zarnowiecki DM, Parfitt G, King S, Dollman J. The experiences of participants in an innovative online resource designed to increase regular walking among rural cancer survivors: a qualitative pilot feasibility study. Support Care Cancer 2014 Jul;22(7):1923-1929. [doi: 10.1007/s00520-014-2177-4] [Medline: 24573604]

28. Hatchett A, Hallam JS, Ford MA. Evaluation of a social cognitive theory-based email intervention designed to influence the physical activity of survivors of breast cancer. Psychooncology 2013 Apr;22(4):829-836. [doi: 10.1002/pon.3082] [Medline: 22573338]

29. Lee MK, Yun YH, Park H, Lee ES, Jung KH, Noh D. A Web-based self-management exercise and diet intervention for breast cancer survivors: pilot randomized controlled trial. Int J Nurs Stud 2014 Dec;51(12):1557-1567. [doi: 10.1016/j.ijnurstu.2014.04.012] [Medline: 24856854]

30. Rabin C, Dunsiger S, Ness KK, Marcus BH. Internet-based physical activity intervention targeting young adult cancer survivors. J Adolesc Young Adult Oncol 2011 Dec;1(4):188-194 [FREE Full text] [doi: 10.1089/jayao.2011.0040] [Medline: 23610737]

31. Valle CG, Tate DF, Mayer DK, Allicock M, Cai J. A randomized trial of a Facebook-based physical activity intervention for young adult cancer survivors. J Cancer Surviv 2013 Sep;7(3):355-368 [FREE Full text] [doi: 10.1007/s11764-013-0279-5] [Medline: 23532799]

32. Kanera IM, Bolman CA, Willems RA, Mesters I, Lechner L. Lifestyle-related effects of the web-based Kanker Nazorg Wijzer (Cancer Aftercare Guide) intervention for cancer survivors: a randomized controlled trial. J Cancer Surviv 2016 Mar 17;10(5):883-897. [doi: 10.1007/s11764-016-0535-6] [Medline: 26984534]

33. Kanera IM, Willems RA, Bolman CA, Mesters I, Verboon P, Lechner L. Long-term effects of a web-based cancer aftercare intervention on moderate physical activity and vegetable consumption among early cancer survivors: a randomized controlled trial. Int J Behav Nutr Phys Act 2017 Feb 10;14(1):19 [FREE Full text] [doi: 10.1186/s12966-017-0474-2] [Medline: 28187725]

34. Valle CG, Deal AM, Tate DF. Preventing weight gain in African American breast cancer survivors using smart scales and activity trackers: a randomized controlled pilot study. J Cancer Surviv 2017 Feb;11(1):133-148. [doi: 10.1007/s11764-016-0571-2] [Medline: 27631874]

35. Block G, Sternfeld B, Block CH, Block TJ, Norris J, Hopkins D, et al. Development of Alive! (A Lifestyle Intervention Via Email), and its effect on health-related quality of life, presenteeism, and other behavioral outcomes: randomized controlled trial. J Med Internet Res 2008;10(4):e43 [FREE Full text] [doi: 10.2196/jmir.1112] [Medline: 19019818]

36. Sternfeld B, Block C, Quesenberry Jr CP, Block TJ, Husson G, Norris JC, et al. Improving diet and physical activity with ALIVE: a worksite randomized trial. Am J Prev Med 2009 Jun;36(6):475-483. [doi: 10.1016/j.amepre.2009.01.036] [Medline: 19460655]

37. Thomas S, Reading J, Shephard RJ. Revision of the physical activity readiness questionnaire (PAR-Q). Can J Sport Sci 1992 Dec;17(4):338-345. [Medline: 1330274]

38. Kushi LH, Doyle C, McCullough M, Rock CL, Demark-Wahnefried W, Bandera EV, American Cancer Society 2010 Nutrition and Physical Activity Guidelines Advisory Committee. American Cancer Society Guidelines on nutrition and physical activity for cancer prevention: reducing the risk of cancer with healthy food choices and physical activity. CA Cancer J Clin 2012;62(1):30-67 [FREE Full text] [doi: 10.3322/caac.20140] [Medline: 22237782]

39. Bandura A. Social cognitive theory: an agentic perspective. Annu Rev Psychol 2001;52:1-26. [doi: 10.1146/annurev.psych.52.1.1] [Medline: 11148297]

40. Bandura A. Human agency in social cognitive theory. Am Psychol 1989 Sep;44(9):1175-1184. [Medline: 2782727] 
41. Locke EA, Latham GP. Building a practically useful theory of goal setting and task motivation. A 35-year odyssey. Am Psychol 2002 Sep;57(9):705-717. [Medline: 12237980]

42. Luca NR, Suggs LS. Theory and model use in social marketing health interventions. J Health Commun 2013;18(1):20-40. [doi: 10.1080/10810730.2012.688243] [Medline: 22934539]

43. Prochaska JO, DiClemente CC. Stages of change in the modification of problem behaviors. Prog Behav Modif 1992;28:183-218. [Medline: 1620663]

44. Ainsworth BE, Irwin ML, Addy CL, Whitt MC, Stolarczyk LM. Moderate physical activity patterns of minority women: the Cross-Cultural Activity Participation Study. J Womens Health Gend Based Med 1999;8(6):805-813. [doi: 10.1089/152460999319129] [Medline: 10495261]

45. Centers for Disease Control and Prevention. CDC. National Health and Nutrition Examination Survey 2008 URL: https:/ /www.cdc.gov/nchs/nhanes.htm [accessed 2017-09-06] [WebCite Cache ID 6tGlSL2Mf]

46. Food and Drug Administration, HHS. Food labeling: trans fatty acids in nutrition labeling, nutrient content claims, and health claims. Final rule. Fed Regist 2003 Jul 11;68(133):41433-41506 [FREE Full text] [Medline: 12856667]

47. U.S. Department of Agriculture ARS. ars.usda. USDA Food and Nutrient Database for Dietary Studies, 2008 URL: https:/ /www.ars.usda.gov/ARSUserFiles/80400530/pdf/fndds/fndds3 doc.pdf [accessed 2017-09-06] [WebCite Cache ID 6tGlnNkXL]

48. Bowen DJ, Kreuter M, Spring B, Cofta-Woerpel L, Linnan L, Weiner D, et al. How we design feasibility studies. Am J Prev Med 2009 May;36(5):452-457 [FREE Full text] [doi: 10.1016/j.amepre.2009.02.002] [Medline: 19362699]

49. Wieland LS, Falzon L, Sciamanna CN, Trudeau KJ, Brodney S, Schwartz JE, et al. Interactive computer-based interventions for weight loss or weight maintenance in overweight or obese people. Cochrane Database Syst Rev 2012;8:CD007675 [FREE Full text] [doi: 10.1002/14651858.CD007675.pub2] [Medline: 22895964]

50. Short CE, Rebar A, James EL, Duncan MJ, Courneya KS, Plotnikoff RC, et al. How do different delivery schedules of tailored web-based physical activity advice for breast cancer survivors influence intervention use and efficacy? J Cancer Surviv 2017 Feb;11(1):80-91. [doi: 10.1007/s11764-016-0565-0] [Medline: 27498099]

51. Short CE, Rebar A, James EL, Duncan MJ, Courneya KS, Plotnikoff RC, et al. How do different delivery schedules of tailored web-based physical activity advice for breast cancer survivors influence intervention use and efficacy? J Cancer Surviv 2017 Feb;11(1):80-91. [doi: 10.1007/s11764-016-0565-0] [Medline: 27498099]

52. Barnett SM, Ceci SJ. When and where do we apply what we learn? A taxonomy for far transfer. Psychol Bull 2002 Jul;128(4):612-637. [Medline: 12081085]

53. Nigg CR, Lee H, Hubbard AE, Min-Sun K. Gateway health behaviors in college students: investigating transfer and compensation effects. J Am Coll Health 2009;58(1):39-44. [doi: 10.3200/JACH.58.1.39-44] [Medline: 19592352]

54. Jayawardene WP, Torabi MR, Lohrmann DK. Exercise in young adulthood with simultaneous and future changes in fruit and vegetable intake. J Am Coll Nutr 2016;35(1):59-67. [doi: 10.1080/07315724.2015.1022268] [Medline: 26251968]

55. Fleig L, Lippke S, Pomp S, Schwarzer R. Intervention effects of exercise self-regulation on physical exercise and eating fruits and vegetables: a longitudinal study in orthopedic and cardiac rehabilitation. Prev Med 2011 Sep;53(3):182-187. [doi: 10.1016/j.ypmed.2011.06.019] [Medline: 21784096]

56. Block G, Azar KM, Block TJ, Romanelli RJ, Carpenter H, Hopkins D, et al. A fully automated diabetes prevention program, Alive-PD: program design and randomized controlled trial protocol. JMIR Res Protoc 2015;4(1):e3 [FREE Full text] [doi: 10.2196/resprot.4046] [Medline: 25608692]

\section{Abbreviations}

ALIVE: A Lifestyle Intervention Via Email

CAPS: Cross-Cultural Activity Participation Study

HRQoL: health-related quality of life

ITT: intention-to-treat

SAS: Statistical Analysis System

SE: standard error

SD: standard deviation

PAR-Q: Physical Activity Readiness Qestionnaire

PAQ: Physical Activity Questionnaire 
Edited by G Eysenbach; submitted 21.02.17; peer-reviewed by L Dean, C Valle; comments to author 26.03.17; revised version received 16.06.17; accepted 27.07.17; published 21.09.17

Please cite as:

Paxton RJ, Hajek R, Newcomb P, Dobhal M, Borra S, Taylor WC, Parra-Medina D, Chang S, Courneya KS, Block G, Block T, Jones $L A$

A Lifestyle Intervention via Email in Minority Breast Cancer Survivors: Randomized Parallel-Group Feasibility Study

JMIR Cancer 2017;3(2):e13

URL: http://cancer.jmir.org/2017/2/e13/

doi: 10.2196/cancer.7495

PMID: 28935620

CRaheem J Paxton, Richard Hajek, Patricia Newcomb, Megha Dobhal, Sujana Borra, Wendell C Taylor, Deborah Parra-Medina, Shine Chang, Kerry S Courneya, Gladys Block, Torin Block, Lovell A Jones. Originally published in JMIR Cancer (http://cancer.jmir.org), 21.09.2017. This is an open-access article distributed under the terms of the Creative Commons Attribution License (https://creativecommons.org/licenses/by/4.0/), which permits unrestricted use, distribution, and reproduction in any medium, provided the original work, first published in JMIR Cancer, is properly cited. The complete bibliographic information, a link to the original publication on http://cancer.jmir.org/, as well as this copyright and license information must be included. 